\title{
Management of Complications Following Emergency and Elective Surgery for Diverticulitis
}

\author{
Christoph Holmer Martin E. Kreis \\ Department of General, Visceral and Vascular Surgery, Charité - Universitätsmedizin Berlin, Campus Benjamin Franklin, Berlin, Germany
}

\section{Keywords}

Sigmoid diverticular disease - Sigmoid resection . Complication management - Anastomotic leakage . Peritonitis

\section{Summary}

Background: The clinical spectrum of sigmoid diverticulitis (SD) varies from asymptomatic diverticulosis to symptomatic disease with potentially fatal complications. Sigmoid colectomy with restoration of continuity has been the prevailing modality for treating acute and recurrent SD, and is often performed as a laparoscopy-assisted procedure. For elective sigmoid colectomy, the postoperative morbidity rate is $15-20 \%$ whereas morbidity rates reach up to $30 \%$ in patients who undergo emergency surgery for perforated SD. Some of the more common and serious surgical complications after sigmoid colectomy are anastomotic leaks and peritonitis, wound infections, small bowel obstruction, postoperative bleeding, and injuries to the urinary tract structures. Regarding the management of complications, it makes no difference whether the complication is a result of an emergency or an elective procedure. Methods: The present work gives an overview of the management of complications in the surgical treatment of SD based on the current literature. Results: To achieve successful management, early diagnosis is mandatory in cases of deviation from the normal postoperative course. If diagnostic procedures fail to deliver a correlate for the clinical situation of the patient, re-laparotomy or re-laparoscopy still remain among the most important diagnostic and/or therapeutic principles in visceral surgery when a patient's clinical status deteriorates. Conclusion: The ability to recognize and successfully manage complications is a crucial part of the surgical treatment of diverticular disease and should be mastered by any surgeon qualified in this field.

\section{Introduction}

Sigmoid diverticulitis (SD) is among the most common diseases in the West; its incidence is rising as the average age of the population increases $[1,2]$. The clinical spectrum of SD varies from asymptomatic diverticulosis to symptomatic disease with potentially fatal complications. Sigmoid resection with restoration of continuity has been the prevailing modality for treating acute and recurrent $\mathrm{SD}$, and is often performed as a laparoscopyassisted procedure [3]. However, laparoscopic resection is not without risk. Although some complications associated with colorectal surgery have been reduced with the use of laparoscopy, including respiratory sequelae, venous thromboembolism, and the incidence of wound infection, other problems such as anastomotic leakage, bowel obstruction, and bleeding persist regardless of the approach used [4-6]. They can lead to significant morbidity and mortality if not recognized early and treated appropriately. For elective sigmoid resection, the postoperative morbidity rate is $15-20 \%$ [7], whereas morbidity rates reach up to $30 \%$ in patients with perforated diverticulitis [8-13]. However, regarding the management of complications, it makes no difference whether the complication is the result of an emergency or an elective procedure. To achieve successful management, early diagnosis is mandatory in cases of deviation from the normal postoperative course. In the following, only the management of common surgical complications is discussed. Anastomotic leakage with peritonitis is the main complication after colorectal surgery and is associated with unfavorable clinical outcomes such as short-term morbidity and mortality. Some more common and serious surgical complications after sigmoid resection are wound infections, small bowel obstruction, bleeding, and urinary tract complications. The present work gives an overview of the management of complications in the surgical treatment of SD based on the current literature.

\section{KARGER \\ Fax +497614520714

() 2015 S. Karger GmbH, Freiburg

$1662-6664 / 15 / 0312-0118 \$ 39.50 / 0$
PD Dr. med. Christoph Holmer 


\section{Complication Management}

\section{General Management}

At each event which deviates from the expected uneventful postoperative course a surgical complication must be excluded first before rare differential diagnoses can be considered. This does not only apply to technically easy interventions, but is particularly important for difficult or technically unsatisfactory surgical procedures. The intraoperative situs is the best indicator of possible postoperative complications [14]. Warning tags or symptoms of postoperative complications always to be considered include persistent or acute pain, fever, paralytic ileus, signs of already disturbed organ functions (e.g. cardiopulmonary, renal), inflammation-related laboratory findings, increase in sepsis parameters, and in particular abnormalities in the drainage secretions (size, aspect, consistency). If these events occur separately or in combination, targeted diagnostics must be initiated immediately.

Sonography is able to specifically identify free fluid or air, and if necessary allows for a sample to be taken by percutaneous puncture or drainage. In addition, sonographic follow-up examinations can be done during conservative treatment at any time. Sonography, however, is technically difficult in the case of major bloating, extreme obesity, major abdominal drainage, and wound or abdominal wall defects.

For computed tomography (CT) the same considerations apply as for ultrasound. As advantages surgeons consider standardized documentation of findings, semi-quantitative density measurement (differential diagnosis: hematoma or abscess), and that an assessment is possible even when sonographic findings are inconclusive.

However, if no morphological correlate for the clinical situation of the patient can be delivered by the specified diagnostic methods, and there is still a high degree of suspicion especially surrounding septic complications, diagnostic re-laparotomy or re-laparoscopy remain as ultima ratio. Nowadays most elective sigmoid colectomies are performed via a laparoscopic approach. Thus re-laparoscopy is a simple and elegant procedure to rule out or pinpoint the presence of a postsurgical complication. The decision to perform re-laparotomy or re-laparoscopy is always quite difficult to make, but a delayed or not performed reoperation could be fatal [15]. To achieve successful management, early diagnosis is mandatory in cases of deviation from the normal postoperative course.

\section{Specific Management}

\section{Anastomotic Leakage}

Anastomotic leaks adversely affect the morbidity and mortality of postoperative patients, may result in a poorer prognosis for functional outcome, and increase the risk of permanent stoma formation. The incidence of anastomotic leakage varies greatly from 1 to $39 \%$, and is dependent on the surgical method and the definition [16-18]. Anastomotic leakage manifests as a spectrum of clinical presentations [19] from obvious peritonism to unspecific signs such as cardiac arrhythmias (e.g. atrial fibrillation) and lower respiratory tract infections. In addition, some anastomotic leaks are manifest only radiologically, occurring in an otherwise asymptomatic patient. In both instances making the diagnosis of anastomotic leakage can prove difficult. Previous studies looking at the diagnosis of anastomotic leaks [20] concluded that significant clinical indicators of leakage were: fever $\left(>38^{\circ} \mathrm{C}\right)$ on day 2 , absence of bowel action on day 4 , diarrhea before day $7,>400 \mathrm{ml}$ of fluid in the abdominal drain by day 3 , renal failure on day 3 , and leukocytosis on day 7. In the study by Khan et al. [21] the median time to leak diagnosis was not until 7 days postoperatively, and our findings also support the significance of fever (median temperature $37.8{ }^{\circ} \mathrm{C}$ ) at this time. The diagnosis of anastomotic leaks may require the use of ultrasound, CT, or contrast studies, but in a subset of patients the decision to reoperate may be driven by clinical aspects alone. The most common investigations performed in the study by Khan et al. [21] were CT scan (41.5\%), Gastrografin ${ }^{\circledR}$ (Bracco Diagnostics Inc., Monroe Township, NJ, USA) enema (14.6\%), and examination under anesthesia (12.2\%). Other authors $[20,22]$ demonstrated that CT scanning with rectal contrast was superior to contrast enemas in the diagnosis of anastomotic leaks following large bowel resections, and was also better at diagnosing intra-abdominal and pelvic abscesses. However, the simplest evidence of intestinal leakage derives from the differentiation of wound secretions and drainage fluids. The diagnosis is secured when intestinal secretion is found to be present in the target drainage either directly or visualized by a marker dye. To achieve successful management, early diagnosis is mandatory in all cases of deviation from the normal postoperative course.

The goals of management are to treat the leak effectively, control sepsis, and convert an urgent or emergent situation into an elective one. The level and intensity of treatment varies with respect to the cause, location, and size of the leak, and the condition and comorbidities of the patient [23].

Stable patients without signs of peritonitis usually can be managed conservatively with the use of drain tubes [24]. This is especially true for smaller anastomotic leaks and existing targeted drainage with complete outward drainage of exudate and without a septic picture. These conditions may be treated conservatively, and spontaneous healing can be awaited. Even larger anastomotic leaks in an extraperitoneal location in the pelvis (typical for anastomoses in the upper third of the rectum after sigmoid resection) can heal secondarily if the anastomosis is protected by a stoma [25].

Large collections are often amenable to percutaneous, transgluteal, or transanal image-guided catheter drainage. The images should be reviewed by an interventional radiologist to identify a safe window of access avoiding vascular structures and other organs. Abscesses of $<3 \mathrm{~cm}$ in diameter will often resolve with a course of antibiotics. In the era of modern CT scanning and interventional radiology, the routine practice of repeat laparotomy, abdominal washouts, large sump drains, and open abdominal wound management is rarely necessary and can be reserved for patients who fail to respond to or deteriorate following percutaneous drainage or who are not considered candidates for this procedure. 
Management of patients with progressive generalized peritonitis with or without septic shock requires balanced rehydration, broad spectrum antibiotics, and urgent laparotomy [23]. Laparoscopic management may be considered if the surgeon has sufficient laparoscopic skills and reoperation experience [26-28]. At the time of surgery the anastomosis should be scrutinized for signs which led to its failure. This will guide the selection of the appropriate method of repair. If the findings include ischemia and necrosis greater than one third of the anastomosis, the anastomosis should be resected and a protective stoma created [21]. When performed in this fashion, subsequent ostomy reversal can be done via a circumstomal incision, obviating the need for formal laparotomy and its associated morbidity. If a smaller leak is identified within an otherwise healthy bowel, the anastomosis can usually be salvaged with suture repair, proximal diversion, and washout of the distal segment [29]. Our preferred diversion is a loop ileostomy. In the case of diffuse peritonitis with septic complication, the Hartmann's procedure should be performed as the safest method.

\section{Peritonitis}

One of the basic principles in the treatment of peritonitis described by Kirschner [30] includes sufficient surgical treatment of the primary source of infection, intraoperative lavage and necrosectomy to reduce bacteria, and definitive abdominal closure with placement of a local drain. An extension of this treatment principle is step-by-step lavage with the aim to clean the abdominal cavity and prevent persistent intra-abdominal infections [31]. A key advantage is the direct control of the initial source of infection and the timely detection of emerging complications due to seam insufficiency, necrotic tissue, and intestinal perforation, all of which can be the starting point of persistent intra-abdominal infections.

Based on improved intensive therapy, highly effective antibiotics, and advances in interventional radiology, a 'wait and see' attitude has become established. $85 \%$ of patients with diffuse peritonitis can be treated successfully with the primary intervention inaugurated by Kirschner alone [30]. Programmed re-laparotomies are not required. Only if patients with maximum intensive therapy and anti-infective treatment do not improve or worsen, a laparotomy 'on demand' should be performed [32].

A randomized trial by Van Ruler et al. [33] showed the advantages of the 'on-demand' approach compared to step-by-step lavage including shorter stay in the intensive care unit, shorter length of hospital stay, and significant cost reduction. For the other competing processes, e.g. closed continuous peritoneal lavage, vacuum treatment, open dorsoventral continuous lavage, and leaving the abdomen open, so far no controlled studies exist due to a lack of comparability of these very heterogeneous patient populations. Thus, a final recommendation is currently not possible [31].

The concept of 'on demand' laparotomy is undoubtedly a more gentle approach for patients favoring faster rehabilitation. The disadvantage is that inspection of the abdominal cavity is not possible during the postoperative course. Thus, postoperative monitoring has to be more subtle and intense. Day by day it must be decided whether surgical eradication of the infectious focus is successful and whether abdominal sepsis is controlled or a laparotomy required. For this decision dilemma, however, no clear concepts exist, and a reliable score of clinical predictors that indicate a persistent abdominal sepsis is to date not available. Hence the decision rests on surgical experience.

If reoperation is needed, this can also be done laparoscopically. Peritonitis should not be considered a contraindication for the laparoscopic approach [34-38]. Current evidence demonstrates that laparoscopy can be successfully used in the emergency treatment of peritonitis from various conditions such as complicated diverticulitis, acute appendicitis, perforated peptic ulcer, or gynecological disease [39]. It has the great advantage of access to all abdominal regions, allowing careful examination of abdominal viscera and thorough cleansing of the peritoneal cavity including deep abdominal areas [39]. However, no definitive recommendations can be made at present based on the existing data.

\section{Wound Infection}

Superficial wound infections are the most common complication of colorectal surgery. Elective colorectal resection in which there is controlled entry into the gastrointestinal tract is classified as a clean-contaminated wound with a predicted incidence of surgical site infection of $5-10 \%$. Risk factors have been identified and include malnutrition, diabetes, immunosuppression, age $>60$ years, American Society of Anesthesia score $>2$, fecal contamination, length of hospitalization before surgery, and extensive surgery [40]. The previously held belief that preoperative cathartic and oral antibiotic bowel preparation is mandatory to prevent postoperative infections has recently been dispelled by multiple randomized controlled trials [41].

Wound infections typically present around the fifth postoperative day and are characterized by any combination of erythema, induration, tenderness, or drainage at the wound site. Systemic signs of fever and tachycardia may also be present. The infection may manifest as an abscess, phlegmon, cellulitis, or a combination of these. If infection is suspected, the wound should be carefully inspected, and when a collection of fluid is detected, it is drained by reopening the wound. Gram stain can assist in management and antibiotic selection. The wound is left open to heal by secondary intention. In most cases cellulitis associated with an abscess will resolve with drainage alone. Antibiotics are usually unnecessary except in cases of cellulitis or an immunosuppressed host.

Necrotizing wound infections are usually the result of either Clostridium perfringens or beta-hemolytic streptococcus, and are potentially life-threatening. These infections generally present within the first few days after surgery and can be difficult to diagnose. Unusually severe wound pain in combination with a thinbodied gray fluid draining from the incision is highly suggestive. These patients should be returned to the operating room for immediate wound exploration and debridement of all devitalized tissue. Broad spectrum antibiotic coverage should include high-dose penicillin. 


\section{Small Bowel Obstruction}

Early postoperative bowel obstruction is rare, occurring in $1 \%$ of patients following intestinal surgery [42]. This time period accounts for $5-29 \%$ of all small bowel obstructions [42-44]. Most obstructions are caused by adhesions which form within $72 \mathrm{~h}$ of surgery, then become very dense and vascularize after 2 weeks. Obstructions are more common following colorectal procedures than following appendectomy or procedures located above the transverse colon. Signs and symptoms of early postoperative small bowel obstruction are similar to (and hard to differentiate from) the more common paralytic ileus. Patients typically develop abdominal distention, nausea, and vomiting, and cannot tolerate nasogastric tube clamping or removal. Most patients have a slow smoldering course with emergency situations being the exception.

There is a fine balance between waiting for the obstruction to resolve and rushing a patient to the operating room. In the first week following surgery, obstruction is hard to differentiate from ileus. Between 2 weeks and 2 months postop, adhesions become thick and vascular and obliterate natural planes, making surgery much more difficult and prone to complications. The decision to operate should therefore occur between 7 and 14 days [43].

Plain films readily diagnose most small bowel obstructions. Oral administration of water-soluble contrast medium followed by an abdominal plain film or CT scan $4 \mathrm{~h}$ later is a good predictor of resolution of a small bowel obstruction. Contrast medium in the colon indicates that the obstruction is likely to resolve with nonsurgical means [45-47]. CT scan may be additionally useful for surgical planning by identifying signs of ischemia and other intraabdominal processes and in localizing the site of obstruction. Management of stable patients involves fluid and electrolyte therapy, bowel rest, nasogastric tube drainage, and nutritional evaluation. Total parenteral nutrition should be started after 7 days. Operation is advised for high-grade or complete bowel obstruction, concern for a strangulated bowel, or unresolved small bowel obstruction despite prolonged nasogastric tube decompression [44]. Most cases resolve with non-surgical management. If surgery becomes necessary, it should occur prior to the 2-week mark after which the acute adhesions become dense, vascular, and problematic. Surgery involves careful re-exploration and lysis of adhesions. Operative findings usually reveal either a single adhesive band or multiple matted adhesions, both modalities occurring with similar frequency. Laparoscopic exploration and adhesiolysis is being increasingly utilized for small bowel obstructions [48]. Advanced laparoscopic skills and experience are a prerequisite. Poor candidates for laparoscopic management include patients with signs of peritonitis, multiple previous operations for small bowel obstruction, small bowel diameter $>4 \mathrm{~cm}$, or other medical contraindications for laparoscopy [49]. Pneumoperitoneum should be established with an open technique at a site remote from the previous incision. Atraumatic graspers are used to explore the bowel in a retrograde fashion beginning with decompressed bowel at the ileocecal valve. Distended bowel is fragile and should not be grasped: grasping the adjacent mesentery reduces the risk of inadvertent bowel perforation. Adhesiolysis is best performed with scissors or bipolar cautery devices to reduce the risk of adjacent bowel injury. Conversion rates range from 7 to $43 \%$ [48]. Proactive reasons to convert include poor visualization, non-viable intestine, multiple dense adhesions, deep pelvic adhesions, and failure to progress within a reasonable amount of time [50].

\section{Bleeding}

Postoperative bleeding can be a life-threatening condition. Patients with early postoperative bleeding $(<48 \mathrm{~h})$ have to be reoperated after sonographic bedside diagnosis without further time-consuming diagnostic procedures. Intraoperative localization of the source of bleeding can be challenging. Careful peritoneal lavage to remove blood and clots, and accurate examination of the trocar sites and the raw surfaces of dissection (commonly the retroperitoneum or pelvis) are required [51]. The combined use of different devices to achieve hemostasis may be useful.

Serious pelvic bleeding during surgery is usually due to injury to the presacral venous plexus or the internal iliac vessels or their branches. Presacral venous bleeding can be difficult to control due to the fragile nature and special anatomy of the presacral venous plexus. Attempts of electrocoagulation or suture ligation may worsen the bleeding and should be avoided. Direct pressure while the patient is being rehydrated by the anesthesia care team is usually the first step. Once stabilized, methods such as sterile thumbtacks, occluded pins, or a rectus muscle patch may be employed [52-54]. The incidence of anastomotic hemorrhage is low (0.5-1\%) [55]. Many anastomotic bleeds are self-limiting and do not require intervention. Distal anastomoses may be localized endoscopically and controlled with injections of dilute epinephrine or short bursts of cautery. Manipulation may increase the incidence of anastomotic leakage. More proximal anastomoses may require operative exploration for control. If suture reinforcement is not effective or if the bleeding source is not obvious, dismantling the anastomosis with resection and re-anastomosis may be required. In the case of late postoperative bleeding ( $>48 \mathrm{~h}$ ), e.g. following septic erosion, angiography provides access to the exact site of bleeding and additionally offers the possibility of simultaneous interventional hemostasis by embolization or bypassing of the corresponding vessel sections with covered stents.

\section{Urinary Tract Complications}

Ureter injuries in colorectal surgery most commonly occur during left-sided colon resections. The following particular steps are at risk during sigmoid resection: ligation of the inferior mesenteric artery and mobilization of the upper mesorectum at the level of the sacral promontory. The key for avoiding a major complication is the intraoperative recognition and repair of a ureteral injury [56]. This may be facilitated by the use of ureteral stents. Indigo Carmi$\mathrm{ne}^{\mathrm{TM}}$ (Akorn Inc., Lake Forest, IL, USA) dye may also be administered if there is suspicion of ureteral injury. The retroperitoneal tissues are then inspected for staining. Repair of ureteral injuries is best performed by a urologist if available. Distal ureteral injuries are best managed by ureteroneocystostomy with or without a vesico-psoas hitch. Mid-ureteral and proximal ureteral injuries can potentially be managed by ureteroureterostomy [57]. 
Bladder injuries are relatively common, especially in the setting of an adherent inflammatory rectosigmoid tumor or diverticular phlegmon [58]. When the bladder wall is either resected or opened, the resulting defect can be closed in two layers with a Foley catheter left in place for 7-10 days postoperatively [59]. A cystogram is often obtained prior to removal of the catheter to confirm healing. Interposition of the omentum between any bladder repair and a bowel anastomosis is advised to prevent fistulization.

\section{Conclusion}

Anastomotic leakage with peritonitis is a rare but very important complication following emergency and elective surgery for SD, and is associated with unfavorable clinical outcomes such as shortterm morbidity and mortality. To achieve successful management, early diagnosis is mandatory in all cases of deviation from the normal postoperative course. If adequate diagnostic methods fail to provide a morphological correlate for the patient's clinical situation, re-laparotomy or re-laparoscopy still remain among the most important diagnostic and/or therapeutic principles in visceral surgery. The goals of anastomotic leakage management are to treat the leak effectively, control sepsis, and convert an urgent or emergent situation into an elective one. In the surgical treatment of peritonitis, early diagnosis of persistent abdominal sepsis and emerging intra-abdominal complications during the course of treatment are crucial for successful treatment.

The ability to recognize and successfully manage complications is a crucial part of surgical treatment of diverticular disease and should be mastered by any surgeon qualified in this field.

\section{Disclosure Statement}

The authors have no conflicts of interest to declare.

\section{References}

1 Jun S, Stollmann N: Epidemiology of diverticular disease. Best Pract Res Clin Gastroenterol 2002;16:529-542.

2 Parks TG: Natural history of diverticular disease of the colon. Clin Gastroenterol 1975;4:53-69.

3 Jacobs M, Verdeja JC, Goldstein HS: Minimally invasive colon resection (laparoscopic colectomy). Surg Laparosc Endosc 1991;1:144-150.

4 Mamidanna R, Burns EM, Bottle A, Aylin P, Stonell C, Hanna GB, Faiz O: Reduced risk of medical morbidity and mortality in patients selected for laparoscopic colorectal resection in England: a population-based study. Arch Surg 2012;147:219-227.

5 Shapiro R, Vogel JD, Kiran RP: Risk of postoperative venous thromboembolism after laparoscopic and open colorectal surgery: an additional benefit of the minimally invasive approach? Dis Colon Rectum 2011;54: 1496-1502.

6 Yamamoto S, Fujita S, Ishiguro S, Akasu T, Moriya Y: Wound infection after a laparoscopic resection for colorectal cancer. Surg Today 2008;38:618-622.

7 Antolovic D, Reissfelder C, Koch M, Mertens B, Schmidt J, Büchler MW, Weitz J: Surgical treatment of sigmoid diverticulitis - analysis of predictive risk factors for postoperative infections, surgical complications, and mortality. Int J Colorectal Dis 2009;24:577-584.

8 Alvarez JA, Baldonedo RF, Bear IG, Otero J, Pire G, Alvarez P, Jorge JI: Presentation, management and outcome of acute sigmoid diverticulitis requiring hospitalization. Dig Surg 2007;24:471-476.

9 Hussain A, Mahmood H, Subhas G, El-Hasani S: Complicated diverticular disease of the colon, do we need to change the classical approach, a retrospective study of 110 patients in southeast England. World J Emerg Surg 2008;3:5.

10 Vermeulen J, Akkersdijk GP, Gosselink MP, Hop WC, Mannaerts GH, van der Harst E, Coene PP, Weidema WF, Lange JF: Outcome after emergency surgery for acute perforated diverticulitis in 200 cases. Dig Surg 2007;24:361-366.

11 Smothers L, Hynan L, Fleming J, Turnage R, Simmang C, Anthony T: Emergency surgery for colon carcinoma. Dis Colon Rectum 2003;46:24-30.
12 Kim J, Mittal R, Konyalian V, King J, Stamos MJ, Kumar RR: Outcome analysis of patients undergoing colorectal resection for emergent and elective indications. Am Surg 2007;73:991-993.

13 McArdle CS, Hole DJ: Emergency presentation of colorectal cancer is associated with poor 5-year survival. $\mathrm{Br}$ J Surg 2004;91:605-609.

14 Siewert JR, Stein HJ, Bartels H: Insuffizienzen nach Anastomosen im Bereich des oberen Gastrointestinaltrakts. Chirurg 2004;75:1063-1070.

15 Hutchins RR, Gunning MP, Lucas DN, Allen-Mersh TG, Soni NC: Relaparotomy for suspected intraperitoneal sepsis after abdominal surgery. World J Surg 2004; 28:137-141.

16 Buchs NC, Gervaz P, Secic M, Bucher P, Mugnier-Konrad B, Morel P: Incidence, consequences, and risk factors for anastomotic dehiscence after colorectal surgery: a prospective monocentric study. Int J Colorectal Dis 2007;23:265-270.

17 Komen N, Dijk JW, Lalmahomed Z, Klop K, Hop W, Kleinrensink GJ, Jeekel H, Ruud Schouten W, Lange JF: After-hours colorectal surgery: a risk factor for anastomotic leakage. Int J Colorectal Dis 2009;24:789-795.

18 Den Dulk M, Noter SL, Hendriks ER, Brouwers MA, van der Vlies $\mathrm{CH}$, Oostenbroek RJ, Menon AG, Steup WH, van de Velde CJ: Improved diagnosis and treatment of anastomotic leakage after colorectal surgery. Eur J Surg Oncol 2009;35:420-426.

19 Alves A, Panis Y, Trancart D, Regimbeau JM, Pocard M, Valleur P: Factors associated with clinically significant anastomotic leakage after large bowel resection: multivariate analysis of 707 patients. World J Surg 2002;26:499-502.

20 Alves A, Panis Y, Pocard M, Regimbeau JM, Valleur P: Management of anastomotic leakage after nondiverted large bowel resection. J Am Coll Surg 1999;189:554-559.

21 Khan AA, Wheeler JM, Cunningham C, George B, Kettlewell M, Mortensen NJ: The management and outcome of anastomotic leaks in colorectal surgery. Colorectal Dis 2008; 10:587-592.

22 Lim M, Akhtar S, Sasapu K, Harris K, Burke D, Sagar P, Finan P: Clinical and subclinical leaks after low colorectal anastomosis: a clinical and radiologic study. Dis Colon Rectum 2006;49:1611-1619.
23 Willis S, Stumpf M: Insuffizienzen nach Eingriffen am unteren Gastrointestinaltrakt. Chirurg 2004;75:10711078.

24 Paliogiannis P, Attene F, Scognamillo F, Trignano E, Torre C, Pulighe F, Trignano M: Conservative management of minor anastomotic leakage after open elective colorectal surgery. Ann Ital Chir 2012;83:25-28.

25 Eckmann C, Kujath P, Schiedeck TH, Shekarriz H, Bruch HP: Anastomotic leakage following anterior resection: results of a standardized diagnostic and therapeutic approach. Int J Colorectal Dis 2004;19:128-133.

26 Rosin D, Zmora O, Khaikin M, Bar Zakai B, Ayalon A, Shabtai M: Laparoscopic management of surgical complications after a recent laparotomy. Surg Endosc 2004; 18:994-996.

27 Kwak JM, Kim SH, Son DN, Kim J, Lee SI, Min BW, Um JW, Moon HY: The role of laparoscopic approach for anastomotic leakage after minimally invasive surgery for colorectal cancer. J Laparoendosc Adv Surg Tech A 2011;21:29-33.

28 Wind J, Koopman AG, van Berge Henegouwen MI, Slors JF, Gouma DJ, Bemelman WA: Laparoscopic reintervention for anastomotic leakage after primary laparoscopic colorectal surgery. Br J Surg 2007;94:1562-1566.

29 Thornton M, Joshi H, Vimalachandran C, Heath R, Carter P, Gur U, Rooney P: Management and outcome of colorectal anastomotic leaks. Int J Colorectal Dis 2011;26:313-320.

30 Kirschner M: Die Behandlung der akuten eitrigen freien Bauchfellentzündung. Langenbecks Arch Chir 1926;142:253-259.

31 Teichmann W, Pohland C, Mausfeld T, Herbig B: Peritonitis: attempt to evaluate therapeutic surgical options. Chirurg 2008;79:282-289.

32 Lamme B, Mahler CW, van Ruler O, Gouma DJ, Reitsma JB, Boermeester MA: Clinical predictors of ongoing infection in secondary peritonitis. World J Surg 2006;30:2170-2181.

33 Van Ruler O, Mahler CW, Boer KR, Reuland EA, Gooszen HG, Opmeer BC, de Graaf PW, Lamme B, Gerhards MF, Steller EP, van Till JW, de Borgie CJ, Gouma DJ, Reitsma JB, Boermeester MA; Dutch Peritonitis Study Group: Comparison of on-demand vs. planned relaparotomy strategy in patients with severe peritonitis: a randomized trial. JAMA 2007;298:865-872. 
34 Agresta F, Ciardo LF, Mazzarolo G, Michelet I, Orsi G, Trentin G, Bedin N: Peritonitis: laparoscopic approach. World J Emerg Surg 2006;1:9.

35 Horattas MC, Haller N, Ricchiuti D: Increased transperitoneal bacterial translocation in laparoscopic surgery. Surg Endosc 2003;17:1464-1467.

36 Ozmen MM, Col C, Aksoy AM, Tekeli FA, Berberoglu $\mathrm{M}$ : Effect of $\mathrm{CO}_{2}$ insufflation on bacteremia and bacterial translocation in an animal model of peritonitis. Surg Endosc 1999;13:801-803.

37 Are C, Talamini MA, Murata K, De Maio A: Carbon dioxide pneumoperitoneum alters acute-phase response induced by lipopolysaccharide. Surg Endosc 2002;16:1464-1467.

38 Montalto AS, Bitto A, Irrera N, Polito F, Rinaldi M, Antonuccio P, Impellizzeri P, Altavilla D, Squadrito F Romeo C: $\mathrm{CO}_{2}$ pneumoperitoneum impact on early liver and lung cytokine expression in a rat model of abdominal sepsis. Surg Endosc 2012;26:984-989.

39 Sauerland S, Agresta F, Bergamaschi R, Borzellino G, Budzynski A, Champault G, Fingerhut A, Isla A, Johansson M, Lundorff P, Navez B, Saad S, Neugebauer EA: Laparoscopy for abdominal emergencies: evidencebased guidelines of the European Association for Endoscopic Surgery. Surg Endosc 2006;20:14-29.

40 Platell C, Hall JC: The prevention of wound infection in patients undergoing colorectal surgery. J Hosp Infect 2001;49:233-238.

41 Guenaga K, Matos D, Castro A, Atallah A, Wille-Jorgensen P: Mechanical bowel preparation for elective colorectal surgery. Cochrane Database Syst Rev 2005; 1:CD001544.
42 Fazio VW, Cohen Z, Fleshman JW, et al: Reduction in adhesive small-bowel obstruction by Seprafilm adhesion barrier after intestinal resection. Dis Colon Rectum 2006;49:1-11.

43 Miller G, Boman J, Shrier I, Gordon PH: Natural history of patients with adhesive small bowel obstruction. Br J Surg 2000;87:1240-1247.

44 Menzies D, Ellis H: Intestinal obstruction from adhesions - how big is the problem? Ann R Coll Surg Engl 1990;72:60-63.

45 Biondo S, Pares D, Mora L, Marti RJ, Kreisler E, Jaurrieta E: Randomized clinical study of gastrografin administration in patients with adhesive small bowel obstruction. Br J Surg 2003;90:542-546.

46 Choi HK, Chu KW, Law WL: Therapeutic value of gastrografin in adhesive small bowel obstruction after unsuccessful conservative treatment: a prospective randomized trial. Ann Surg 2002;236:1-6.

47 Chen SC, Lin FY, Lee PH, Yu SC, Wang SM, Chang KJ: Water-soluble contrast study predicts the need for early surgery in adhesive small bowel obstruction. Br J Surg 1998;85:1692-1694.

48 Wullstein C, Gross E: Laparoscopic compared with conventional treatment of acute adhesive small bowel obstruction. Br J Surg 2003;90:1147-1151.

49 Zerey M, Sechrist CW, Kercher KW, Sing RF, Matthews BD, Heniford BT: Laparoscopic management of adhesive small bowel obstruction. Am Surg 2007;73: 773-778.

50 Borzellino G, Tasselli S, Zerman G, Pedrazzani C, Manzoni G: Laparoscopic approach to postoperative adhesive obstruction. Surg Endosc 2004;18:686-690.
51 McCormick JT, Simmang CL: Reoperation following minimally invasive surgery: are the 'rules' different? Clin Colon Rectal Surg 2006;19:217-222.

52 Nivatvongs S, Fang DT: The use of thumbtacks to stop massive presacral hemorrhage. Dis Colon Rectum 1986;29:589-590.

53 Stolfi VM, Milsom JW, Lavery IC, Oakley JR, Church JM, Fazio VW: Newly designed occluder pin for presacral hemorrhage. Dis Colon Rectum 1992;35:166-169.

54 Remzi FH, Oncel M, Fazio VW: Muscle tamponade to control presacral venous bleeding: report of two cases. Dis Colon Rectum 2002;45:1109-1111.

55 Dochetry JG, McGregor JR, Akyol AM, et al: Comparison of manually constructed and stapled anastomoses in colorectal surgery. Ann Surg 1995;221:176-184.

56 Halabi WJ, Jafari MD, Nguyen VQ, Carmichael JC, Mills S, Pigazzi A, Stamos MJ: Ureteral injuries in colorectal surgery: an analysis of trends, outcomes, and risk factors over a 10-year period in the United States. Dis Colon Rectum 2014;57:179-186.

57 Burks FN, Santucci RA: Management of iatrogenic ureteral injury. Ther Adv Urol 2014;6:115-124.

58 Ostrzenski A, Ostrzenska KM: Bladder injury during laparoscopic surgery. Obstet Gynecol Surv 1998;53: 175-180.

59 Elliott SP, McAninch JW: Ureteral injuries: external and iatrogenic. Urol Clin North Am 2006;33:55-66. 